\title{
Computational Chemistry Methods for Nanoporous Materials
}

\author{
Jack D. Evans, ${ }^{\dagger}$ Guillaume Fraux, ${ }^{\dagger}$ Romain Gaillac, ${ }^{\dagger,}$ Daniela Kohen, ${ }^{\dagger, \S}$ Fabien Trousselet, ${ }^{\dagger}$ \\ Jean-Mathieu Vanson, ${ }^{\dagger, \|, \perp}$ and François-Xavier Coudert* ${ }^{*} \dagger$ \\ ${ }^{\dagger}$ Chimie ParisTech, PSL Research University, CNRS, Institut de Recherche de Chimie Paris, 75005 Paris, France \\ ${ }^{\ddagger}$ Air Liquide, Centre de Recherche Paris Saclay, 78354 Jouy-en-Josas, France \\ ${ }^{\S}$ Department of Chemistry, Carleton College, Northfield, Minnesota 55057, United States \\ "École Normale Supérieure, PSL Research University, Département de Chimie, Sorbonne Universités - UPMC Univ Paris 06, CNRS \\ UMR 8640 PASTEUR, 24 rue Lhomond, 75005 Paris, France \\ ${ }^{\perp}$ Laboratoire de Synthèse et Fonctionnalisation des Céramiques, UMR 3080 Saint Gobain CREE/CNRS, 550 Avenue Alphonse \\ Jauffret, 84306 Cavaillon, France
}

\section{Supporting Information}

ABSTRACT: We present here the computational chemistry methods our group uses to investigate the physical and chemical properties of nanoporous materials and adsorbed fluids. We highlight the multiple time and length scales at which these properties can be examined and discuss the computational tools relevant to each scale. Furthermore, we include the key points to consider-upsides, downsides, and possible pitfalls-for these methods.

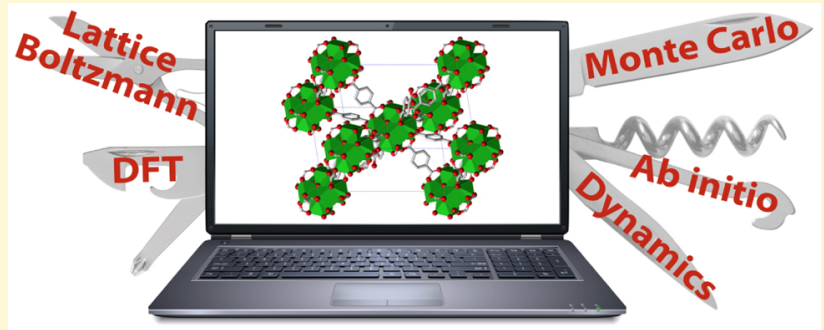

\section{INTRODUCTION}

The Coudert research group uses molecular simulation, at various scales, to study a wide range of porous materials and molecular fluids near interfaces or under confinement. Outlined herein is a review of the computational chemistry toolbox we routinely use. Nonetheless we will first say here a few words about the systems of interest to our research: nanoporous materials and the adsorption of molecular fluids within their pore space.

Nanoporous materials exhibit high internal surface area, resulting in a wide range of applications, examples of which include large-scale processes in key sectors of the chemical industry, such as gas separation and capture, ${ }^{1}$ storage ${ }^{2}$ liquid separation, heterogeneous catalysis, ${ }^{3}$ drug delivery, ${ }^{4}$ and more. ${ }^{5}$ Specifically, nanoporous materials display a very rich range of chemical composition (inorganic, organic, metal-organic) and geometrical properties (macroporous, mesoporous, microporous). This offers a diverse scope of physical and chemical properties. Among nanoporous materials, our group focuses on two main families of materials, namely zeolites and metalorganic frameworks (MOFs). ${ }^{6,7}$ Inorganic zeolites represent the current generation of porous materials widely used in industrial processes, due to their large-scale availability, reasonable cost, and high thermal, mechanical, and chemical stability. The very topical metal-organic frameworks (MOFs) are a more recent class of materials. MOFs are widely considered contenders for specific applications, due to their extraordinarily high porosity and tunability of both their framework dimensions and internal surface chemistry. Of particular interest to our group are framework materials that exhibit counterintuitive or "anom- alous" physical properties, such as stimuli-responsive materials (sometimes also called multifunctional or "smart" materials). ${ }^{8}$ These frameworks exhibit large-scale changes in their structure and physicochemical properties in response to small stimuli, such as temperature change, mechanical constraint, guest adsorption, and exposure to light or magnetic fields.

Our research seeks to capture the structure, dynamics, and thermodynamics of nanoporous systems using an atomistic level description. Because the systems vary widely in length scales, and the processes we want to observe can significantly differ in their time scales, we use different "levels of theory" or theories with different assumptions. This may, for example, include explicitly treating the electrons of each atom ("quantum chemistry" approaches) ${ }^{9}$ or treating atoms or groups of atoms as a single unit ("classical" approaches). ${ }^{10}$ In all cases, however, the goal of computational chemistry is the same: to model the macroscopic behavior of complex heterogeneous systems, constructed from individual entities and their interactions, under certain external (experimental) conditions. This is achieved by relying on statistical thermodynamics as our fundamental tool for bridging the interactions at the atomistic scale and the macroscopic properties of a condensed matter system. Though numerical methods of statistical thermodynamics can be applied to nonequilibrium processes, ${ }^{11}$ we focus

Special Issue: Methods and Protocols in Materials Chemistry

Received: July 21, 2016

Revised: September 3, 2016

Published: September 6, 2016 
here on computational methods to study systems in thermal, mechanical, or chemical equilibrium.

For a system in thermodynamic equilibrium in a given experimental condition, its state is determined by a number of thermodynamic variables forming a thermodynamic ensemble. An isochoric-isothermal process, for example, takes place in the commonly used $(N, V, T)$ canonical ensemble, in which the quantity of matter $(N)$, system volume $(V)$, and absolute temperature $(T)$ are fixed. In a given ensemble, the thermodynamics of the system and its macroscopic properties are then entirely determined by the statistical distribution of all possible microscopic states (or microstates) of the system. This is formalized through the ensemble's partition function, whose simplest expression is, for a system with quantized states in the canonical ensemble and with classical Maxwell-Boltzmann statistics:

$$
Z(N, V, T)=\sum_{i} \exp \left(\frac{-E_{i}}{k_{\mathrm{B}} T}\right)
$$

where $i$ is the microstate index of the system, $k_{B}$ is Boltzmann's constant, and $E_{i}$ is the total energy of a given microstate.

In practice, all molecular simulation methods have the same goal: to evaluate the macroscopic properties of the system by averaging microscopic properties over a collection of microstates $i$ (this is the $\sum_{i}$ part of eq 1 ), weighted by the respective probability of occurrence (Boltzmann probability), which depends on their energy (the individual $E_{i}$ 's involved in the summation). The differences between the various computational approaches available are due to different methods of evaluating the energy of states and of sampling the microstates of the system.

Methods of evaluating energy in these systems can use complex quantum mechanical descriptions including HartreeFock, post-Hartree-Fock, and the ubiquitous density functional theory (DFT). These highly accurate methods can elucidate structural and mechanical properties resulting from electronic processes. Alternatively, energies can be calculated using classical potentials, which are less computationally demanding than quantum mechanical approaches. However, classical simulations require accurate potential parameters (force fields) and cannot straightforwardly model quantum phenomena such as changes in chemical bonding.

Additionally, numerical simulation is used in the exploration of states as illustrated in Figure $1 \mathrm{~b}$. A number of different methods can be employed to result in an energy or dynamic motion of the structure. Most simply, a single state can be evaluated to obtain a single-point energy of the system; however, more commonly, we seek to obtain an optimal structure (local energy minimum) or transition states. Minima, and other states, are realized by iterative single-point energy evaluation corresponding to changes of the structure. Moreover, through molecular dynamics, the motion of structures is generated by an exploration of states using iterative integration of Newton's laws of motion. Finally, states can be explored by stochastic displacements-this is the basis for Monte Carlo simulations, a useful method for simulating gas adsorption.

A number of properties, or time and length scales, are not available by direct molecular simulation as described above. Here we use several geometric and statistical physics based approaches at the mesoscale. The combination of all these methods is a rather powerful computational chemistry toolbox for nanoporous materials, that we highlight in this review.
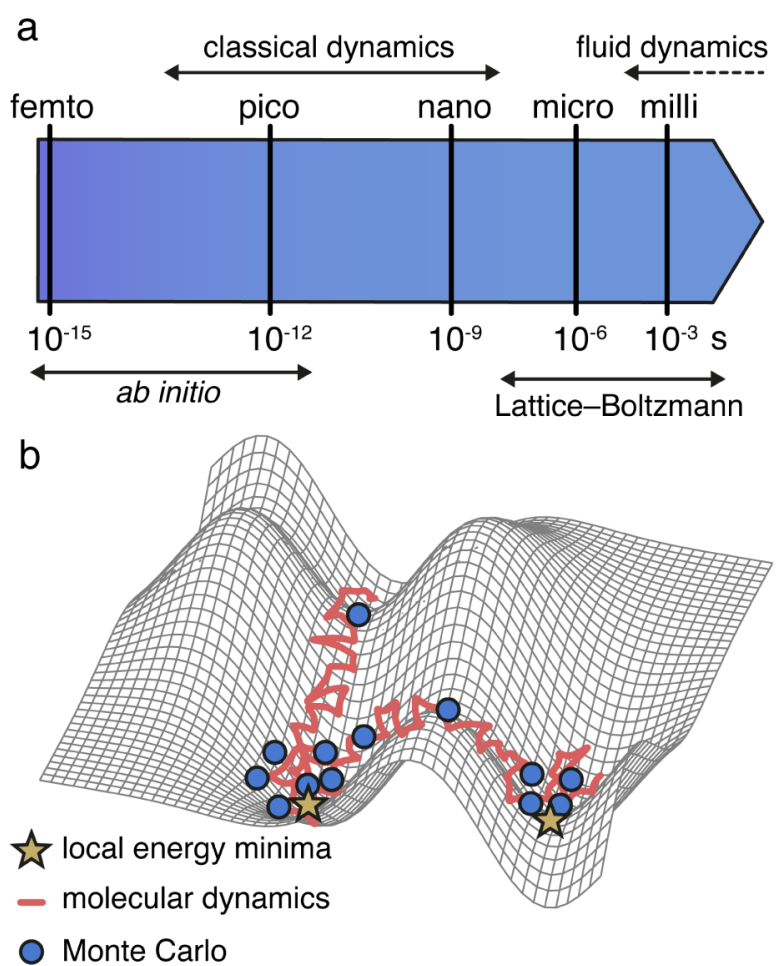

Figure 1. Depiction of time scales captured by the simulation approaches used in our research (a) and alternative simulation methods for exploring a chemical energy surface (b).

\section{QUANTUM MECHANICAL SIMULATION}

In order to study the structure, energetics, electronic states, and other physicochemical properties of porous crystals with a high level of accuracy, one of the tools we routinely use is quantum chemistry calculations based on density functional theory (DFT). ${ }^{9}$

Fundamentally, in quantum simulations of electronic groundstate properties, the wave function has to be solved $(\psi)$ to satisfy the Schrödinger equation to give the energy $(E)$ of the system. An example of the Schrödinger equation for multiple electrons interacting with multiple fixed nuclei is given in eqs 2 , 3 , and 4 . In these equations $m$ is the electron mass and the Hamiltonian $(\hat{H})$ has terms relating to the kinetic energy of each electron $(\hat{T})$, the interaction energy between the atomic nuclei and each electron $(\hat{V})$, and finally the interaction energy between different electrons $(i$ and $j$ ) for $N$ electrons $(\hat{U})$.

$$
\begin{aligned}
& \hat{H} \psi=E \psi \\
& {[\hat{T}+\hat{V}+\hat{U}] \psi=E \psi} \\
& {\left[-\frac{\hbar^{2}}{2 m} \sum_{i=1}^{N} \nabla_{i}^{2}+\sum_{i=1}^{N} V\left(\mathbf{r}_{i}\right)+\sum_{i=1}^{N} \sum_{j<i} U\left(\mathbf{r}_{i}, \mathbf{r}_{j}\right)\right] \psi=E \psi}
\end{aligned}
$$

The complete wave function is complicated, even for a simple molecule, owing to each atom having many electrons. For instance, the full electronic wave function for $\mathrm{CO}_{2}$ is a 66dimensional function. Furthermore, the electron-electron interaction term of the Hamiltonian $\left(\sum_{i=1}^{N} \sum_{j<i} U\left(\boldsymbol{r}_{i}, \boldsymbol{r}_{j}\right)\right)$ is crucial and requires considering the correlations of each individual electron with all the other electrons. Therefore, the wave function of the system is a complicated function that 
makes an exact or analytical solution impractical. Notably, wave functions cannot be directly observed; however, eq 5 illustrates how the density of electrons $(n(r))$ is determined from the individual electron wave functions, $\psi_{i}$.

$$
n(\mathbf{r})=2 \sum_{i} \psi_{i}^{*}(\mathbf{r}) \psi_{i}(\mathbf{r})
$$

DFT methods apply two Hohenberg-Kohn theorems to reduce the complexity of the problem immensely. ${ }^{12}$ The first states that the ground-state energy (and other properties) is determined uniquely by the ground-state electron density. As a consequence, a solution to the Schrödinger equation requires a function of three spatial coordinates, the electron density, rather than the original wave function, which has $3 \mathrm{~N}$ variables for $N$ electrons. The second states that the correct electron density minimizes the overall energy functional and subsequently corresponds to the solution of the Schrödinger equation. This latter theorem affords a direction toward finding the electron density of the system. Kohn and Sham applied these theorems to show the correct electron density can be determined by solving a set of single electron equations of the form illustrated in eq $6 .{ }^{13}$ The solutions of these equations are straightforward single-electron wave functions that depend on three spatial coordinates.

$$
\left[-\frac{\hbar^{2}}{2 m} \nabla^{2}+V(\mathbf{r})+V_{\mathrm{H}}(\mathbf{r})+V_{\mathrm{XC}}(\mathbf{r})\right] \psi_{i}(\mathbf{r})=\varepsilon_{i} \psi_{i}(\mathbf{r})
$$

The Kohn-Sham equations contain three potentials: $V, V_{\mathrm{H}}$ and $V_{\mathrm{XC}}$. The first, $V$, describes the interaction between an electron and the collection of atomic nuclei, similar to $\hat{V}$. This potential has a simple analytical form. The second, $V_{\mathrm{H}}$, describes the repulsion between the single-electron and the total electron density. It is named the Hartree potential and is defined by eq 7, where the electron density is given by $n$. Importantly, this potential includes a self-interaction contribution, as the electron described by the Kohn-Sham equation also contributes to the total electron density. Thus, $V_{\mathrm{H}}$ includes a nonphysical Coulombic interaction concerning an electron and itself which is corrected for in $V_{\mathrm{XC}}$. This final potential, $V_{\mathrm{XC}}$, characterizes the electron exchange and correlation interactions to the single-electron Kohn-Sham equations. The potential is formally defined as a "functional derivative" of the exchange-correlation energy $\left(E_{\mathrm{XC}}\right)$ which is not known, except for a free electron gas. Nevertheless, approximations exist, which permit the calculation of certain physical quantities accurately.

$$
V_{\mathrm{H}}(\mathbf{r})=e^{2} \int \frac{n\left(\mathbf{r}^{\prime}\right)}{\left|\mathbf{r}-\mathbf{r}^{\prime}\right|} \mathrm{d}^{3} r^{\prime}
$$

To solve the Kohn-Sham equations, the definition of Hartree potential $\left(V_{\mathrm{H}}\right)$ requires the electron density $(n)$, which depends on the single-electron wave functions $\left(\psi_{i}\right)$, which in turn requires the solution of the Kohn-Sham equations. As such, an iterative approach is applied to obtain a solution. ${ }^{14}$ This is routinely achieved using the self-consistent field method, an example of a simplified algorithm for this approach is outlined below:

1. Define an initial trial electron density, $n(r)$.

2. Calculate corresponding potentials, $V, V_{H}$, and $V_{\mathrm{XC}}$, for the electron density.

3. Solve the Kohn-Sham equations to find the singleelectron wave functions, $\psi_{i}$.
4. Calculate the electron density, $n_{\mathrm{KS}}$, from the constructed Kohn-Sham equations using the single-electron wave functions, $\psi_{i}$, from step 3 .

5. Compare the calculated electron density, $n_{\mathrm{KS}}(\boldsymbol{r})$, with the electron density used in solving the Kohn-Sham equations, $n(r)$. If the two densities are the same, then this is the ground-state electron density and it can be used to compute the total energy. If the two densities are different, then a new trial electron density, $n(\boldsymbol{r})$, is used and the process begins again from step 2 .

Reliable approximations for the exchange-correlation functional $\left(E_{\mathrm{XC}}\right)$ are required for the solution of the Kohn-Sham equations. There are a number of approaches for treating exchange-correlation, including local density approximation (LDA), generalized-gradient approximation (GGA), metaGGA, and hybrid functionals. Each functional has particular strengths and weaknesses that must be considered and proven.

Ultimately, the power of DFT is simplifying a $3 N$ dimensional problem, where $N$ is the number of electrons, into a 3-dimensional problem. Consequently, DFT is a popular choice for electronic structure calculation, of both molecules and crystals, because of the accuracy obtained for relatively cheap computational cost. Moreover, it is readily available and widely implemented in several open source, academic, and commercial software packages. Recently, by comparing the equation of states for 71 elemental crystals with many DFT codes and methods, a collection of the solid state community has demonstrated the reproducibility of DFT simulations. ${ }^{15}$ The results for modern codes and methods agree very well, exhibiting pairwise differences which are comparable to different experiments conducted with high-precision. In the following, we show how we use DFT to predict theoretical structures of new materials, model mechanical responses by elastic constant calculations, and even study the dynamics of materials on short time scales. Moreover, we describe how we typically perform these calculations, and which technical aspects are important for numerical efficiency and quality of results.

Modeling Crystals with DFT. The simplest type of DFT calculation on crystal structures are "static" DFT calculations used to explore local features of their energy landscape. This includes single-point energy calculations, energy minimization, harmonic vibration modes, and more. Since most of our systems (MOFs and zeolites) typically feature high symmetry, we employ the CRYSTAL14 software package. ${ }^{16}$ CRYSTAL14 uses localized basis sets and is appropriate for insulating systems, such as zeolites and MOFs. This methodology is computationally efficient in porous systems, compared to plane waves which would describe void and occupied spaces with the same level of precision. In practice, atomic basis sets are finite and their size (and parametrization), for each element in the system, is chosen as a compromise between computational cost and accuracy. Depending on the accuracy needed, we use either the DZVP (double- $\zeta$ valence polarized) or TZVP (triple- $\zeta$ valence polarized) basis sets, or alternatively basis sets parametrized for chemically similar compounds. Importantly, there is a detailed library of basis sets cataloged by the software developers, available online at http://www.crystal.unito.it/basis-sets.php. To determine which type of basis set to use, we usually run single-point energy calculations with the various candidate basis sets, and check the quality of the description of the electronic states obtained. This is typically done by comparing values of electron density, atomic partial charges, and band gap to our 
chemical knowledge of the system, or higher-level quantum chemical calculations.

For DFT calculations, the level of approximation of electronic exchange and correlation effects $\left(E_{\mathrm{XC}}\right)$ is the main cause of approximation (or inaccuracy). Therefore, the choice of exchange-correlation functional has a significant influence on how the energy depends on atomic positions, and thus on all calculated properties. While many options are available in the literature and implemented in popular software, the decision on exchange-correlation functional has to be guided by a compromise between accuracy and computational cost. Welike many others-typically use generalized-gradient approximation (GGA) functionals to take into account the nonlocal exchange and correlation effects, which is not possible with local density approximation (LDA) functionals. Furthermore, when computationally possible, we employ hybrid functionals which provide a better description of the electronic exchange. This is achieved by adding exact Hartree-Fock exchange, mixed with exchange and correlation terms computed at the GGA level.

Generally, the appropriate functional is contingent on the property of interest. In practice, we have found that the PBE functional revised for solids (PBESOL) ${ }^{17}$ in addition to a hybrid version, PBE0 (PBESOL0), produce consistent and physical results in many porous systems. ${ }^{18,19}$ Another common choice of hybrid functional in the literature is B3LYP: ${ }^{20}$ although it is usually considered more appropriate for molecular calculations than in the solid state, it has been widely used in MOFs and appears to give reliable results overall. In cases of zeolites, which are built from stronger interactions than MOFs, we note that most properties (structure, energetics, mechanics) are well described already with GGA functionals such as PBESOL. However, for simulations of complex properties, such as band structures, the use of hybrid functionals is preferred. ${ }^{21}$

It is well-known, and should be noted here, that DFT is quite poor at representing long-range correlation effects, typically including dispersion interactions. When required, dispersion correction terms are added to the functional using the D2 correction scheme detailed by Grimme and co-workers, ${ }^{22}$ implemented in the CRYSTAL14 software. The D2 correction is a relatively simple function of interatomic distances. This includes adjustable parameters fitted to interaction and conformational energies computed using high-level wave function methods. Subsequently, this correction is added to the energy obtained by DFT and, as such, does not directly modify electron density. Notably, there are a number of alternative dispersion correction methods, ${ }^{23}$ such as D3 and the many-body-dispersion (MBD) scheme. ${ }^{24,25}$ Dispersion corrections are often essential, and we have found they can strongly influence energetic and mechanical properties, especially for porous structures. Specifically, in MOF systems, dispersion corrections often improve structural agreement with experimental crystal structures-although counter-examples can be found because Grimme's simple and empirical "D2" correction scheme can lead to overestimation of the dispersive interactions at intermediate intermolecular distances $(6-10 \AA)$. Thus, for each system, we benchmark the performance of both approaches, without and with dispersion, to experimental data: in particular, we consider the crystal density, unit cell parameters, and key intermolecular distances $(\pi-\pi$ stacking, hydrogen bonds, etc.), crucial quantities typically affected by dispersive interactions.
Another important consideration for DFT calculations of crystals is the choice of reciprocal space sampling. This is controlled by a shrinking factor, used to generate a corresponding grid of $k$-points in reciprocal space. For structures with very large unit cells, reciprocal space sampling can be limited to the $\Gamma$ point; in general, it should be chosen so as to reach convergence on single-point energy. Recently, the importance of $k$-point convergence has been clearly described in simulations of MIL- $47(\mathrm{~V}) .^{26}$ A rule of thumb we often use, as a starting point, is to have the smallest lattice parameter multiplied by the value of the shrinking parameter be around 20-30 A. In some structures with very asymmetric cells, for example MIL-47, ${ }^{27}$ we use direction-dependent shrinking parameters, to refine the mesh in specific directions. Within Crystal14, the space group of the system (when known) is considered to reduce the computational cost by reducing the number of spatial integrals to compute, ideally by the order of the space group. This can be very efficient: for example the MOF structure UiO-66, with a cubic cell and space group $F \overline{4} 3 m$, and point group symmetries, reduces the CPU time by a factor of approximately 16 (for a single-point energy calculation on 12 processors) (Figure 2 ).

Finally, we note that a simple and useful way to supervise DFT calculations is to examine, during the self-consistent field (SCF) iteration scheme for the calculation of the density and energy, the atomic partial charges (calculated by the software with the Mulliken scheme). Because we have a reasonable chemical intuition of these quantities, they provide an initial and rapid feedback as to the quality of the density calculation: when the $\mathrm{Si}$ atoms in a zeolite acquire a net charge of 15 , the simulation is certainly diverging! Subsequently, if convergence seems unreachable, with standard minimization schemes and parameters, one can modify the initial population of orbitals for the first SCF cycle as to increase the damping of the SCF algorithm, to achieve self-consistence. Alternatively, the Broyden scheme for self-consistent field calculations is very robust. $^{28,29}$ Mulliken population analysis, commonly used to obtain atomic partial charges, has explicit basis set dependence $^{30}$ and may not display good accuracy. As atomic partial charges are nonphysical quantities, there are complications and subjectivity to calculation of these values. Thus, there are a number of alternative methods which are more suited to accurately describing porous systems. ${ }^{31}$ However, to simply verify calculations, we find the Mulliken method is adequate.

The quantum mechanical simulations conducted by our group primarily use the CRYSTAL14 software, which is unique in treating periodic systems with a crystalline local orbital method. However, we note many groups employ simulation codes, such as Quantum Espresso ${ }^{33}$ and VASP, ${ }^{34}$ which use a plane-wave approach. This method implicitly treats periodicity in the description of the wave function. While both approaches have particular advantages and disadvantages, for example, planewave methods require a large number of basis functions (augmenting significantly memory requirements) and crystalline local orbitals do not efficiently treat low symmetry systems. $^{35}$ In particular, researchers have employed planewave approaches to investigate many nanoporous material properties, including the reactivity of zeolite catalysts ${ }^{36}$ and electrical conductivity in MOFs. ${ }^{37}$

Calculation of Structural and Mechanical Properties. We highlight here the typical output we obtain from DFT calculations of porous crystals. For further detail, one can find 
a

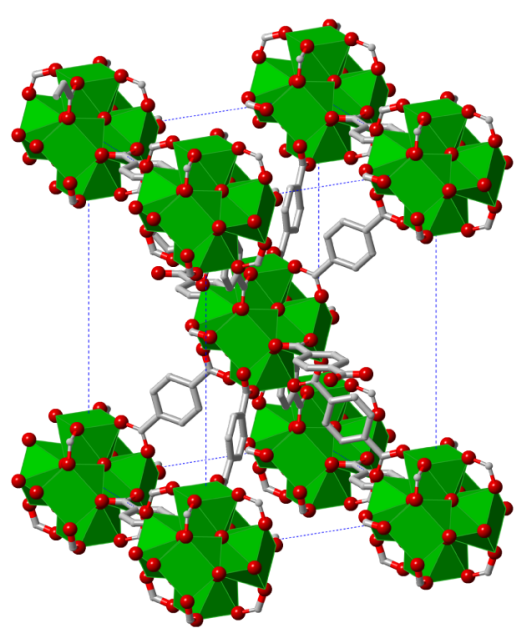

b

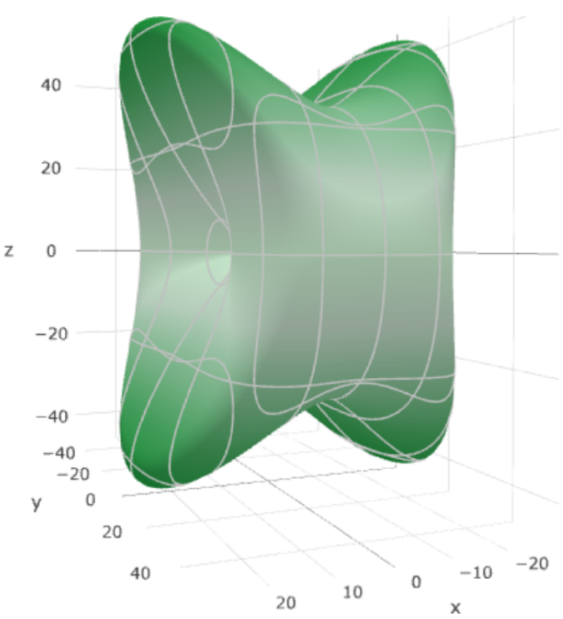

Figure 2. An example of the use of density functional theory (DFT) calculations for porous materials: calculation of (a) the structure of a defective $\mathrm{UiO}-66(\mathrm{Zr})$ MOF with partial substitution of benzene dicarboxylate linkers by formate capping ligands; ${ }^{18}(\mathrm{~b})$ its directional Young's modulus, in units of GPa, plotted by the ELATE software ${ }^{32}$ from the DFT-computed elastic constants.

comprehensive reviews on the many properties calculated using DFT simulation. ${ }^{38-40}$

The most common result-and arguably the most crucial result-of DFT calculations on porous crystals is the optimized structure, including cell parameters and atomic positions. Geometry optimizations (CRYSTAL14 keyword OPTGEOM) consist of iteratively updating the structure and subsequently computing energies and forces. Notably, initial structures are chosen from known structures such as crystallographic data; otherwise, several plausible structures are considered to sample the phase space. This iterative procedure is repeated until convergence criteria are met, i.e. until atomic displacements and forces are smaller than a chosen threshold. To determine the direction and amplitude of each step (in the $\mathbf{R}^{3 N}$ space of atomic coordinates, with $N$ the number of atoms per cell), we generally use a numerical quasi-Newton method, the BroydenFletcher-Goldfarb-Shanno algorithm. We use the convergence criteria standard in CRYSTAL14 $\left(9 \times 10^{-4} \mathrm{Ry} / \mathrm{bohr}\right.$ on each component of the energy gradient). Occasionally tighter criteria are used to ensure higher accuracy, typically for properties linked to derivatives of the energy (vibration modes or mechanical properties, see below). The number of time steps needed to reach convergence can vary between 10 and 20 , typical for small, high-symmetry systems with rather smooth energy landscapes. However, this can be $>200$ in problematic cases, or if the initial structure is obviously far from optimal. This occurs if simulations begin with a poorly resolved experimental structure or atoms are in unrealistic proximity. Furthermore, as optimizations are constrained to the space group symmetry, we often relax space group requirements and perturb the system. Consequently, we ensure a minimum of lower energy, with a lower symmetry, cannot be found and the space group constraint is appropriate.

In the case of mechanical properties calculations (keyword ELASTCON), the main output is the $6 \times 6$ tensor of secondorder elastic constants $C_{i j}$ in Voigt notation. These are computed by performing small deformations along each of the 6 deformation modes, or a subset of those in high symmetry systems (2 modes are enough for cubic space groups). We then analyze it further with tensor analysis tools ${ }^{41}$ such as the ELATE web application ${ }^{32}$ (http://progs.coudert. name/elate) developed in our group to investigate the mechanical properties of the material studied, including both volumetric and anisotropic properties. The primary properties are the bulk modulus, in addition to direction-dependent Young's moduli, linear compressibilities, shear moduli, and Poisson's ratios. ${ }^{18,42}$ These calculations are generally more costly than geometry optimizations, with the computation time depending not only on the system size but also on a number of other factors: the number of deformation modes to be performed, from 2 in cubic systems to 6 in triclinic systems; the point group symmetries remaining after individual deformations (for an orthorhombic system, shearing modes take much more time than compression modes, which do not break any symmetry); and the number of steps needed to reach convergence, after each cell deformation, which can be decreased by decreasing the deformation amplitude. We have included a representative input file, used for mechanical property simulations with CRYSTAL14, in the Supporting Information.

Aside from elastic constants, we also use DFT calculations to analyze vibrational modes, examining frequencies and mode eigenvectors. Periodic structures have a number of quantized modes of vibration, in which the lattice uniformly oscillates. These normal vibrational modes, also called phonons, influence a number of physical properties, such as thermal and electrical conductivity. ${ }^{43}$ The starting point, for these simulations, must be a properly optimized structure, a local minimum in the space of atomic coordinates. A standard approach, generally sufficient for systems with large unit cells (cell parameters $\geq 15 \AA$ ) is to compute harmonic vibrations (keyword FREQCALC), where all atoms with identical fractional coordinates in neighboring cells vibrate in phase. Specifically, these calculations allow for the estimation of the contribution of vibrations to the entropy as described in eq 8 , where $\beta=1 / k_{B} T{ }^{44}$ This description can be generalized to other thermodynamic quantities, including heat capacity. In addition, we estimate Grüneisen parameters, which in turn give access to the thermal expansion coefficient. ${ }^{45}$

$$
S_{\text {vib }}=k_{\mathrm{B}} \sum_{i}^{3 N}\left[-\ln \left(1-\mathrm{e}^{-\beta \varepsilon_{i}}\right)+\frac{\beta \varepsilon_{i}}{\mathrm{e}^{-\beta \varepsilon_{i}}-1}\right]
$$

Vibrational mode calculations consists of $3 \mathrm{~N}$ independent steps (or usually less, thanks to symmetry relations). At each step, one atomic coordinate is slightly modified (typically by 0.3 
$\mathrm{pm}$ ) and the resulting energy variation and forces are computed taking into account a possible lowering of symmetry. Even for high-symmetry systems, these computations can be computationally quite demanding since most deformations break most symmetries; for UiO-66, ${ }^{46}$ while a single-point and force calculation takes about 4 min (using 12 processors), the whole calculation of vibration frequencies takes about $11 \mathrm{~h}$, performing only 18 deformations. For systems with small unit cells, it is safer to compute phonon frequencies on a finite number of points of the Brillouin zone (keywords SCELPHONO and DISPERSI), rather than merely at the $\Gamma$ point. Commonly, vibrational calculations result in identification of imaginary frequencies, a nonphysical result and indication the structure is not a local minima. To address such spurious frequencies, we suggest increasing the DFT convergence to extremely highprecision, such that frequencies may be more accurately assigned to zero or positive frequencies. However, this may also be an indication that the initial configuration was not well optimized.

These types of simulations are usually tractable for several hundreds of atoms per unit cell on 16 cores. Except for specific cases, the parallelization becomes inefficient when exceeding 20 to 30 processors. For example, when the shrinking parameter is higher, using more cores becomes more efficient. These simulations can be run on a desktop workstation with more than 16 cores, local computing facilities, or national highperformance computing (HPC) centers. The largest system ever treated in the group, at the national HPC scale, is the metal-organic framework DUT-49 with 1728 atoms in a conventional cell of $46.7 \AA$ parameter (cubic space group $\mathrm{Pa} \overline{3}$, 72 atoms in the asymmetric unit). ${ }^{47}$

$\boldsymbol{A} \boldsymbol{b}$ Initio Molecular Dynamics. In addition to the study of structures and "local" properties (often called "zero Kelvin" properties, because they do not account for thermal motions and entropy), the finite-temperature motion of condensed matter systems can be examined at the quantum chemical level using $a b$ initio molecular dynamics (AIMD). Although much less frequent than local DFT calculations because of its much higher computational cost, we consider AIMD to be an essential tool to probe dynamical properties in situations where subnanosecond dynamics are relevant. It is applicable to all condensed matter systems, not limited to crystals but also including interfaces and complex liquids, as demonstrated in a recent study of the transport mechanism of carbon dioxide in molten carbonates. ${ }^{48}$ We highlight here our use of AIMD to study MOFs and zeolitic frameworks as well as porous materials where adsorbates are present, using the $\mathrm{CP} 2 \mathrm{~K}$ simulation package - a general open source quantum chemistry and solid state physics simulation package, available at https:// www.cp2k.org.

The idea behind molecular dynamics (MD) is to explore the dynamic motion of molecules by numerically solving Newton's equations of motion:

$$
\mathbf{F}_{i}\left(r^{N}\right)=-\nabla_{i} U\left(r^{N}\right)=m_{i} \frac{\mathrm{d}^{2} r_{i}}{\mathrm{~d} t^{2}}
$$

The force, $\mathbf{F}_{i}$ experienced by an atom $i$ of mass $m_{i}$ and position $r_{i}$ is equal to the negative of the gradient, $\nabla_{i}$, of the potential energy of the system defined by electron density, $U\left(r^{N}\right)$. Subsequently, Newton's second law relates the force to the particle's mass multiplied by the second derivative of its position, $r_{i}$, with respect to time, $t$, i.e. the particle's acceleration.
In $a b$ initio $\mathrm{MD}$, the atomic forces are calculated at the quantum chemical level, typically using DFT. CP2K uses the Gaussian and plane wave (GPW) method or a Gaussian and augmented plane wave (GAPW) method to accurately describe the electronic density within the system. ${ }^{49}$ VandeVondele et al. have reported a complete and thorough description of these methods used by CP2K and other codes. ${ }^{50,51}$

The motion of the nuclei follows Newton's equation, and is integrated numerically with a finite size time step $\Delta t$. At each time step the wave function is optimized in the DFT framework, thus combining the accuracy of $a b$ initio calculations and the ability to study time dependent phenomena. This method is thus much more costly that static calculations, but can be used to study mechanisms where bond cleavage and bond formation are essential or where no classical approximation (force field) of the intra- and intermolecular interactions is available.

Simulations in several different thermodynamic ensembles can be performed: constant number of particles, volume, and temperature $(N, V, T)$; constant number of particles, pressure, and temperature $(N, P, T)$. Moreover, the simulation of rare events such as chemical reactions and infrequent diffusion using AIMD is challenging. By combining AIMD with constrained dynamics and metadynamics methods, we and other researchers have been able to elucidate properties relating to rare events with high accuracy. ${ }^{52,53}$ The output from the simulations is typically the system's wave function-although it is costly to store on disk due to its large size-and population charges on each atom (and for each time step). AIMD simulations primarily result in the positions, the velocities, and the forces on each atom of the system as well as the instantaneous temperature, stress tensor, and, for $(N, P, T)$ simulations, cell parameters.

By analysis of the resulting trajectories we can calculate a number of properties. First, pair distribution functions (PDFs) which, when all atoms are taken into account, can be compared with PDF from X-ray diffraction analyses. This is useful for validating simulations. By examining individual PDFs, we are able to identify the relevant interactions, which is not possible from experimental data. Moreover, statistical analyses are used to quantify events such as bond cleavage and formation; specifically, this was used to study the mechanism of pyrocarbonate formation and its separation into carbon dioxide and carbonate anion. ${ }^{48}$ Additionally, computing coordination numbers aids in monitoring the stability of coordination networks. ${ }^{54}$ Vibrational modes within the framework or in the presence of a guest molecule can be obtained by effective normal-mode analysis, ${ }^{55}$ and preferred sites of adsorption are found by simple analysis of the spatial distribution of adsorbates. Extraction of fundamental free energy profiles is achieved by identifying a potential of mean force or from more sophisticated approaches using constraint molecular dynamics such as the Blue Moon Ensemble approach. ${ }^{56,57}$ These methods are quite powerful; for example, we have recently used them to uncover the mechanism by which a carbon dioxide molecule can permeate a zeolite where cations block narrow pores for other gases (Figure 3).

Importantly, these methods are quite costly in terms of computational power. For simulations in the $(N, V, T)$ ensemble of MOFs with approximately 300 atoms, we can simulate between 2000 and $6000 \mathrm{fs}$ in $10 \mathrm{~h}$ using 256 cores on national high-performance computing systems. Simulations in the $(N, P, T)$ ensemble are often three times slower than 


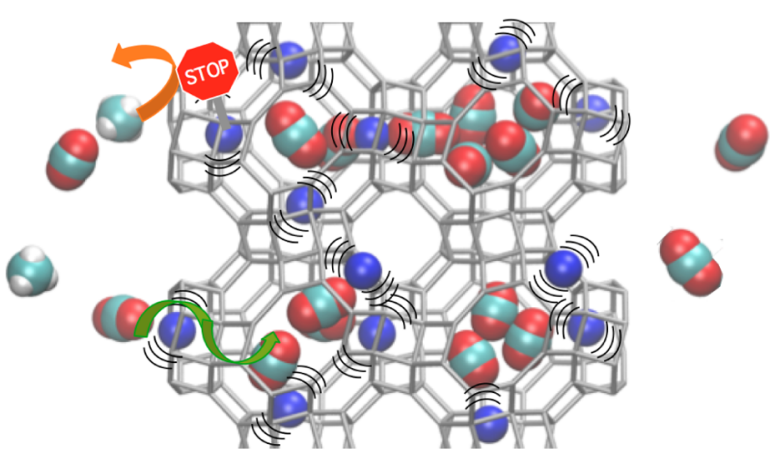

Figure 3. AIMD simulations allowed us to uncover the mechanism by which a carbon dioxide molecule can permeate a zeolite where cations block narrow pores for other gases by taking advantage of cations thermal motion. ${ }^{52}$ In gray: Na-RHO zeolitic framework; blue: $\mathrm{Na}^{+}$ cations; $\mathrm{CO}_{2}$ (cyan and red) guest molecules can bypass the sodium ions and diffuse through the zeolite's windows, while methane (cyan and white) cannot.

$(N, V, T)$ simulations, as the temperature and pressure converge much slower. Despite this, we were able to accurately describe two structurally different phases of a very flexible MOF. ${ }^{58}$ In particular, massive parallelization is not possible for this type of calculation; specifically, an increase from 64 to 128 cores has an efficiency of about $40 \%$, and from 128 to 256 cores about $50 \%$, but increasing from 256 to 512 cores can even make the calculation $20 \%$ slower. Scaling studies are crucial before running simulations in order to be computationally efficient, or at least not to waste computational resources.

During simulations, we check that the chosen parameters allow us to represent the system correctly. Conservation of energy and other conserved quantities are essential, in addition to convergence of temperature, as it shows that the thermostat has the desired effect, and the same goes for the pressure (keeping in mind that pressure fluctuations on such small systems can be very large $(\sim 10000$ bar for a volume of 4000 $\left.\AA^{3}\right)$ ). We also check that the cell parameters and the charges remain plausible and they do not diverge or converge to obviously nonphysical values. The number of self-consistent field steps needed to converge the energy at each time step is also a good hint of whether or not the input parameters, such as the time step, have been well-chosen. It is also crucial to check that no unrealistic phenomena occur, such as a zeolite framework breaking covalent bonds in usual conditions of temperature and pressure.

Additionally, we have attached a representative input file for an MD simulation in the Supporting Information. This corresponds to a $\mathrm{MD}$ run for zeolite $\mathrm{Na}-\mathrm{RHO}$ with carbon dioxide adsorbed.

\section{CLASSICAL SIMULATION}

Herein we have shown the wealth of information obtainable by quantum mechanical simulation; however, for longer time scales and larger systems, classical molecular simulations are employed. Typically, classical simulations can be performed routinely on systems containing thousands of atoms, or in more extreme cases for systems up to 100000 atoms $^{59}$-although due to their periodic nature, it is not common in simulations of crystalline materials. Simulation times in classical dynamics can also be much larger that in $a b$ initio $\mathrm{MD}$, ranging from a few nanoseconds to hundreds of nanoseconds. This allows molecular level insight into macroscopic material properties such as adsorption, diffusion, and framework dynamics. ${ }^{60-62}$

Force Field Parameters. Potential energy $\left(U\left(r^{N}\right)\right)$ in classical simulations is calculated from an interatomic potential energy function that is described by parameters from the force field. Importantly, the choice and implementation of force fields is crucial to the accuracy obtained by this method.

Force fields are parametrized such as to reproduce the molecular geometry or thermodynamic properties reported experimentally or described by higher level $a b$ initio calculations. The general functional form of the potential energy function in classical molecular simulation includes bonded terms for interactions of atoms that are linked by covalent bonds (or metal-ligand bonds), and nonbonded or noncovalent terms that describe long-range electrostatic and van der Waals forces illustrated in Figure 4. The specific

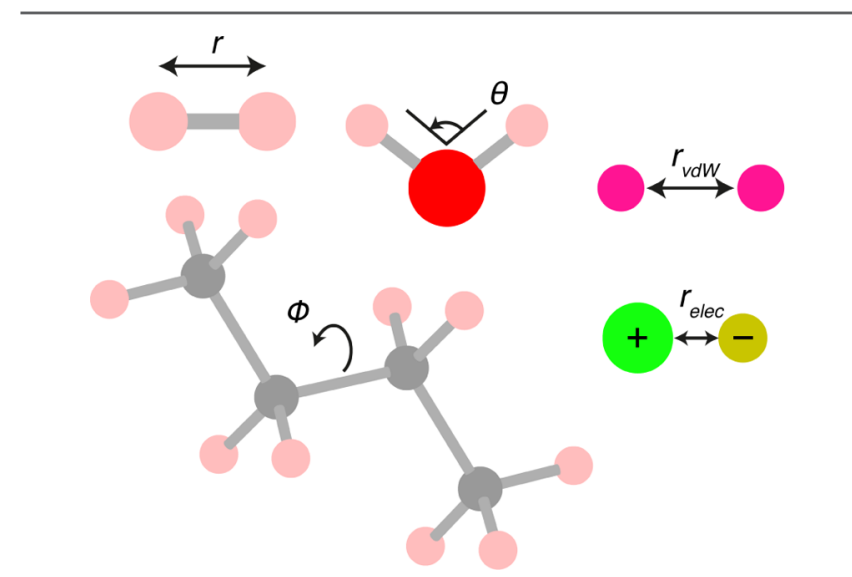

Figure 4. Examples of the structural parameters explicitly described by force field models used in classical molecular simulations.

decomposition of the terms depends on the force field applied, but a general form for the total energy in an additive force field is illustrated in eqs 10,11 , and 12 .

$$
\begin{aligned}
& U_{\text {total }}=U_{\text {bonded }}+U_{\text {nonbonded }} \\
& U_{\text {bonded }}=U_{\text {bond }}+U_{\text {angle }}+U_{\text {dihedral }} \\
& U_{\text {nonbonded }}=U_{\text {electrostatic }}+U_{\text {van der Waals }}
\end{aligned}
$$

There are a great variety of options for force fields, with early examples developed to reproduce the geometry of small organic molecules and later adapted to treat more complex functionalized molecules. ${ }^{63}$ Moreover, there are a number of general force fields that have been developed to treat all atoms in the periodic table, ${ }^{64}$ biomolecules, ${ }^{65}$ and condensed matter. ${ }^{66}$ Simulations employing these general force fields have been shown to be applicable to many porous systems; in particular, universal force field (UFF) ${ }^{64}$ parameters have been ubiquitous in calculating intermolecular energies $\left(U_{\text {van der Waals }}\right)$ for gas adsorption simulations. ${ }^{67}$

Force fields for porous structures with a high degree of flexibility require good accuracy in the description of intramolecular interactions in the structure, in particular for the low-frequency phonon modes present, which is often not captured by generic force fields. ${ }^{68}$ To this end, researchers have used $a b$ initio simulation to derive accurate system specific force fields. van Speybroeck and co-workers have developed QuickFF for the easy derivation of new force fields from $a b$ initio simulations, ${ }^{69}$ which has been used to describe a number of 
flexible MOFs and explore thermodynamics. ${ }^{70}$ Alternatively, Heine et al. have extended UFF for use in MOF systems by revising and adding parameters relating to common transition metal nodes. ${ }^{71}$ Nevertheless, the choice of force field applied in classical simulation must be initially tested against experimental or quantum chemistry methods to demonstrate the accuracy of the method.

Methodologies and Implementation. Though classical simulation, like quantum mechanical simulation, can be used to obtain single-point energies and local minima, we primarily use classical methods to investigate the dynamics of molecules and adsorption processes.

Molecular dynamics is achieved by the same iterative process described previously for AIMD. However, owing to the cheaper computational costs of classical simulation, we can follow processes on a larger scale, such as water confined in zeolites. ${ }^{72}$ In addition to the choice of force field, as discussed previously, the options of time step and thermostat (or in the case of $(N, P$, $T$ ) simulations thermostat and barostat) are crucial. For greatest computational efficiency, the time step should be chosen as large as possible such to minimize the number of calculation steps for a given time period. However, the time step must be verified, so that it does not result in drifts or large fluctuations in energy and other conserved quantities. Notably, this is also true for AIMD simulations. Moreover, molecular dynamics explicitly represents the $(N, V, E)$ ensemble; thus, to compare to experimental systems, dynamic constraints (thermostat and barostat), applied to the movement or particles or cell parameters, are added. This results in a fixed averaged value of temperature or pressure resulting in the $(N$, $V, T)$ and $(N, P, T)$ ensembles. There are many approaches to fixing these averages, each with particular advantages and disadvantages that must be considered before application. ${ }^{74,75}$ In particular, we routinely use the Nosé-Hoover thermostat and barostat. ${ }^{76-78}$ When using this method, there is a choice of relaxation time, which defines the time scale for the relaxation of temperature or pressure. We often choose relaxation times of $1-5 \mathrm{ps}$, ensuring the temperature and pressure do not fluctuate significantly and the equilibration time is not unnecessarily long.

The adsorption process can be simulated by Monte Carlo (MC) simulations which consist of trial displacement, insertion, and removal of gas molecules in the framework structure. ${ }^{79}$ The criteria for accepting a trial displacement are often described by the Metropolis algorithm. ${ }^{80}$ In the Metropolis algorithm in a simple application, the below steps are followed until a move is accepted.

1. Calculate the potential energy $\left(U_{i}\right)$ of the initial state.

2. Choose a trial displacement of a random molecule from a uniform random distribution.

3. Calculate the potential energy of the new state $\left(U_{f}\right)$.

4. If $U_{f} \leq U_{i}$, accept the move.

5. If $U_{f}>U_{i}$, select a random number, $w$, where $w \in[0,1]$ from a uniform distribution and if $\exp \left[-\beta\left(U_{f}-U_{i}\right)\right]>w$, accept the move.

6. Repeat steps 2-5.

In grand-canonical Monte Carlo, the number of molecules adsorbed in the porous structure evolves over the course of the simulation, and after a period of equilibration, it will fluctuate around an equilibrium value imitating the experimental system, in which the adsorbed phase is at equilibrium with a gas reservoir. Conducting Monte Carlo simulations can be very straightforward; however, there are a number of important options and analyses to consider before conducting a simulation, including the relative probability of different trial moves (rotation, displacement, reinsertion, and more) and ensuring convergence in energy or number of adsorbed particles. Dubbeldam et al. have produced an excellent resource for the application of MC in this area. ${ }^{79}$

In particular, we have employed classical molecular dynamics to examine the stability of 18 zeolitic imidazolate frameworks (ZIFs), displayed in Figure $5 .^{81}$ By using a classical approach,
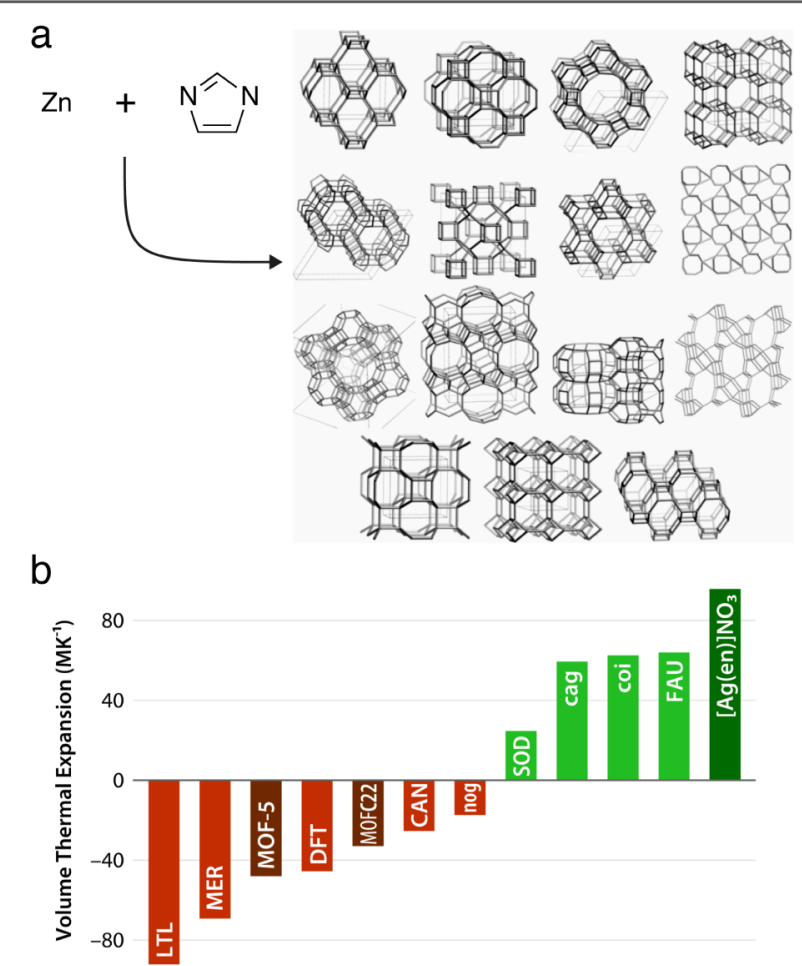

Figure 5. Components of zeolitic imidazolate frameworks (ZIFs) and their possible topologies examined (a). Resulting volume thermal expansion coefficients, simulated by classical molecular dynamics, for the ZIF frameworks (b). This is compared to values reported in the literature for other metal-organic frameworks, portrayed in a darker shade. This figure was adapted from ref 81 .

we were able to simulate the framework dynamics of the 18 frameworks at multiple temperatures for more than $5 \mathrm{~ns}$, a scale not computationally feasible using $a b$ initio methods. Unit cell fluctuations were measured in response to changes in temperature and pressure to quantify the relative stability of the topologies. Notably, we observed a number of the examined ZIFs displaying significant negative thermal expansion (Figure 5 ), and many of the included hypothetical topologies are not mechanically stable at reasonable temperatures.

As computational chemists, our findings rely on software implementations of the simulation algorithms described briefly above. The two main categories of software used in simulation are small, homegrown software that are used at the scale of one or two research groups, and very big software, used by hundreds (if not thousands) of researchers. Most of the classical molecular dynamics codes around belong to the second category, such as LAMMPS, ${ }^{82}$ GROMACS, ${ }^{83}$ and DLPOLY. $^{84}$ This situation arose mainly because of the complexity of the algorithms used in molecular dynamics, where very-largescale systems are simulated on supercomputers, and significant 
development effort is required to get an efficient implementation. On the other hand, most publicly available MC codes, such as RASPA ${ }^{85}$ and many others, are homegrown software. This can be explained by the diversity of the algorithms, and the capacity to tailor a specific MC move for a specific system. For example, the GIBBS code ${ }^{86}$ was initially developed for the simulation of hydrocarbons in zeolites, and as such proposes specific MC moves such as chain reptation, partial rotation of molecules, and biased insertion/deletion of chain molecules.

Sometimes, the existing software does not provide what we need for our studies. For example, the coupling between adsorption and deformation of the guest framework is hard to study with the current methods. This coupling is at the origin of the complex and fascinating behaviors of some MOF, including breathing, ${ }^{87}$ gate-opening, ${ }^{88}$ or more complex behaviors. ${ }^{47}$ The natural ensemble for simulating these phenomena is the osmotic ensemble, ${ }^{89}$ which is an extension of the grand-canonical ensemble. Molecular dynamics cannot be used easily for simulations in the grand-canonical ensemble, because most of the algorithms rely on an Hamiltonian dynamic, which is hard to obtain with a varying number of particles. (Some schemes have been proposed for grandcanonical molecular dynamics, ${ }^{90,91}$ but it is not yet clear whether the ensemble simulated using these scheme is the grand-canonical ensemble or not.) On the other hand, MC methods can easily sample the grand-canonical ensemble, but have a lot more difficulties to reproduce large amplitude and collective movements, which naturally occur in these phenomena. One solution to get efficient sampling of the osmotic ensemble is to use a hybrid Monte-Carlo simulation, where short runs of molecular dynamics are used as MC moves.

Unfortunately, hybrid MC simulations are not implemented in any of the current widely used molecular simulation software, and implementing such a feature in existing codes would necessitate large flexibility of the code base. ${ }^{92}$ As a result, we have begun to write a new simulation code, with the explicit goals of being modular and to ease the new methods. We aim to make this code easy to read and to modify, and reasonably fast. At the time of writing, the code is able to perform energy minimization, molecular dynamics simulations in the $(N, V, E)$, $(N, V, T)$, and $(N, P, T)$ ensemble, MC simulations in the $(N$, $V, T),(N, P, T)$, grand-canonical ensemble, and hybrid MC simulation in all ensembles, including the osmotic ensemble. All these simulation methods share the same implementation of energy evaluation, including Ewald sum and Wolf sum for the treatment of electrostatic interactions. ${ }^{93,94}$ The software is undergoing a cleaning phase before we share it openly with the community.

Currently the development of scientific software is often overlooked, with software bugs that either give wrong results or prevent reproducibility. ${ }^{95}$ To improve software quality and maintainability, scientists can and should use proven techniques from software engineering. ${ }^{96}$ These techniques are moderns answers to an old problem: humans are fallible, how do we make sure that the code is correct? Methods we use for the development of our in-house software include version control using git ${ }^{97}$ and Github; ${ }^{98}$ unit tests, which check the behavior of one function only; and integration tests, which test that multiple parts of the code work together (for example, is the energy constant during a molecular dynamics simulation, do we get the pressure of the system right in $(N, P, T) \mathrm{MC})$. All these tests are run each time new code is written, using multiple compilers and operating systems. Services like Travis ${ }^{99}$ provide server farms for free for open-source projects to build and run tests using Linux or OS X and various compilers (GCC and clang). Additional code review ensures that the code is read and understood by different persons, catching some additional mistakes. Together, theses methods help to ensure the reproducibility of the simulations and improve the usability of the resulting software by removing a lot of bugs.

\section{MESOSCALE SIMULATION}

We have shown how characterization at the quantum and atomistic scale can elucidate interesting phenomena exhibited by porous materials. However, there are limits to the efficiency and system size accessible by these methods. To achieve examination of the mesoscopic properties of porosity and largescale transport processes, we use a number of tools which take further approximations to the chemistry structure.

Pore Structure Identification. Surface area and pore metrics are vital characteristics of porous materials, particularly for applications in gas storage and separation, where the performance of materials is often correlated with these quantities. ${ }^{100}$ Notably, MOFs are reported to have very high surface areas, over $7000 \mathrm{~m}^{2} \mathrm{~g}^{-1} ;{ }^{101}$ however, surface area cannot be measured directly from experiments. The surface area of a porous material is commonly obtained by application of Brunauer-Emmett-Teller (BET) theory to Ar or $\mathrm{N}_{2}$ adsorption isotherms. ${ }^{102,103}$ The BET method relies upon a number of assumptions: adsorption occurs on a homogeneous surface, no lateral interactions occur between adsorbed molecules, and Langmuir theory is applicable to each adsorbed layer. While for a number of cases these assumptions may not hold true, ${ }^{104}$ and while such surface area measurements give no microscopic insight into the nature of the porosity, being a standard analysis technique, they offer a widely used, simple, and reproducible characterization tool for nanoporous materials.

There are a number of algorithms and tools that are used to obtain pore metrics from a well-defined periodic structure. For the most direct method, the surface area and pore volume can be calculated geometrically from the crystal structure. ${ }^{105}$ Popular through application in the Accelrys Materials Studio, the surface area of a material corresponds to the area traced out by the center of a probe particle as the probe particle is moved across the surface of the framework atoms, illustrated in Figure 6a. These accessible areas calculated with a probe molecule equivalent to the size of nitrogen are in good agreement with the experimental BET surface areas-although exceptions can arise because of the dynamic nature of the framework, or pore blocking by guest molecules due to incomplete activation of the experimental samples. Alternatively, there are a number of more complex methods to describe the detailed porosity of a periodic system including Poreblazer, ${ }^{106}$ ZEOMICS, ${ }^{107}$ and Zeo++. ${ }^{108}$ Each of these methods can identify the pore size distribution, maximum pore size, and pore limiting diameter to result in a clear picture of the system's pore structure. An example of the pore metrics obtainable using Zeo++ is represented in Figure $6 \mathrm{~b}$.

The methods outlined here provide a crucial role of bridging the microscopic structure, elucidated by quantum mechanical or classical simulation, to the surface area or porosity observed experimentally.

Fluid Flow and Adsorption. Understanding the interplay between transport of species and adsorption in porous materials, at a mesoscopic scale, is one of the main steps to 
a
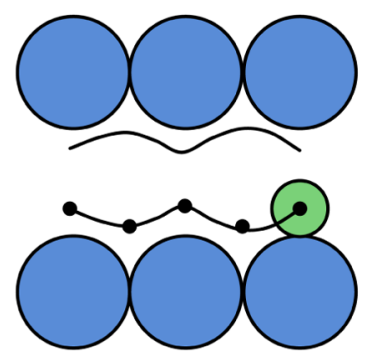

framework atom

\section{— pore surface}

b
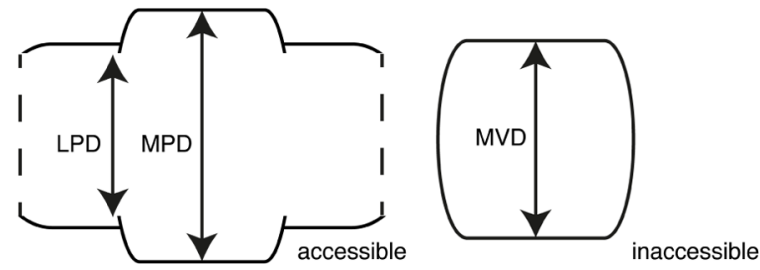

Figure 6. Representation of the geometric method used to measure the pore volume and surface area in porous materials (a). Additionally, the pore information obtained by software such as Zeo++, limiting pore diameter (LPD), and maximum pore diameters (MPD) for pore channels and maximum void diameters (MVD) can be found for inaccessible voids present within the structure (b).

improve the efficiency of porous materials for applications such as ion capture, ion exchange, and phase separation. The number of atoms or molecules needed to perform simulations with molecular dynamics or Monte Carlo models at large length scales (micrometer) makes atomistic approaches computationally unfeasible. Macroscopic methods used in classical computational fluid dynamics can be used to simulate fluid behavior, but lack in their description of local phenomena, such as adsorption. In between these two scales, there exist a few mesoscale lattice-based methods, although they are much less commonly used than atomistic simulations and computational fluid dynamics. In our group, in order to computationally study liquid flow and adsorption in porous materials, we use a Lattice-Boltzmann (LB) model recently expanded to account for adsorption. ${ }^{109}$ Its basic principles are illustrated in Figure 7. The Lattice-Boltzmann scheme is a computational fluid dynamic (CFD) model working on a 3D mesh of equally spaced nodes, i.e. a grid of cubic voxels. It allows us to compute efficiently the fluid behavior in the material. Physical quantities related to the fluid flow (such as the fluid velocity field) and the adsorption (concentration of adsorbed and free species) are stored for each node of the mesh. Then, instead of solving the Navier-Stokes equations as is done in standard CFD methods, these fields are numerically integrated in time through a discrete formulation of the Boltzmann transport equation. The algorithm we are using here is based on the scheme summarized by Ladd and Verberg. ${ }^{110}$ For a given external force, equivalent to a drop pressure, it computes the flux and density field of fluid. Here we investigate mesoporous materials, and the regime is assumed to be linear with a no slip boundary condition at the interface solid/liquid.

The LB model finds its roots in the lattice gas cellular automata method and statistical physics with the introduction of a Boltzmann equation. ${ }^{111-113}$ It works on the propagation of a single particle velocity distribution function $f(r, c, t)$ representing the probability of one particle to be at position $r$ with the velocity $c$ at time $t$. This function is propagated on the lattice using the discrete velocity model D3Q19 (3 dimensions, 19
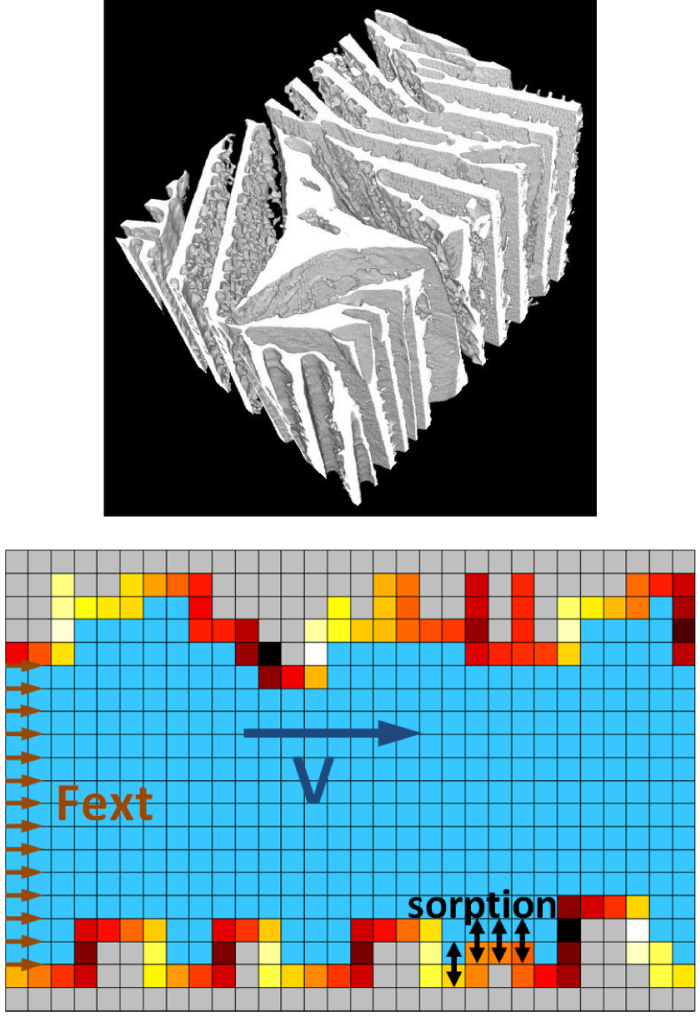

Figure 7.2D graphic representation of the Lattice-Boltzmann scheme expanded to account for adsorption. The solid part of the material is in gray, the liquid part is in blue, and the colored gradients show the adsorbed density. The figure below is a 3D binarized image of a porous material obtained with X-ray tomography we may use as input for our simulations.

speeds). Other velocity models are available, such as the $\mathrm{D} 3 \mathrm{Q} 15$ or the D3Q27, but the D3Q19 offers the best compromise between speed and precision. ${ }^{114}$ The dynamics of the LB scheme follows this propagation described in eq 13, where $f_{i}^{e}$ represents the local Maxwell-Boltzmann equilibrium distribution, $\tau$ is the relaxation time, and $F_{i}^{\text {ext }}$ accounts for external forces. For a better understanding of the fundamentals of the LB model, refer to the work of Succi. ${ }^{115}$

$$
\begin{aligned}
& f_{i}\left(r+c_{i} \Delta t, t+\Delta t\right) \\
& \quad=f_{i}(r, t)+\frac{\left(f_{i}^{e}(r, t)-f_{i}(r, t)\right)}{\tau}+F_{i}^{e x t}
\end{aligned}
$$

The LB model is coupled with a moment propagation method $^{116,117}$ simulating the motion of species called "tracers" inside the fluid. The adsorption part of the algorithm, taking place at the solid/liquid interface, is introduced in the moment propagation method. ${ }^{109}$ It has been recently improved to account for saturation of the tracers on the adsorption sites and thus reproduce a Langmuir adsorption type. ${ }^{118}$ The dispersion coefficient, the diffusion coefficient, the quantity adsorbed, and the density adsorbed are computed in this part. This coefficients give interesting information about the motion of tracers inside the fluid.

The LB scheme employed here has the particularity to be adimensional. Inputs and output values are expressed as a function of the distance between nodes and the time step. We use an internal code to create input files and compute results. This code includes a library of typical geometries automatically 
generated, such as slit pore, cylinder, honeycomb, and inverse opal.

X-ray tomography imaging (see Figure 7) can be easily used to create an input of real materials with a simple binarization process. The definition of the lattice (3D cubic grid) of the $\mathrm{LB}$ scheme corresponds exactly to the voxel definition of a 3D image. This type of geometry gives a powerful tool to simulate flow and adsorption in real materials.

The code is parallelized using OpenMP. We simulate geometries up to $64 \times 10^{6}$ nodes on conventional multithreaded computers. In the case of small geometries (1001000 nodes), we run a simulation on 4 to 8 cores. For big geometries ( $>1,000,000$ nodes $)$ we run simulations on 20 to 30 cores. We usually use convergence criteria on the mean fluid velocity, fraction adsorbed, velocity autocorrelation function, and dispersion coefficient. The dispersion coefficient is computed from the velocity autocorrelation function. However, we always consider carefully the convergence of the dispersion coefficient because a good convergence on the velocity autocorrelation function does not necessarily imply a convergence of the dispersion coefficient. A representative input file used for LB simulations is demonstrated in the Supporting Information.

\section{CONCLUSION}

Computational chemistry is a useful toolbox for understanding the very large diversity of physical and chemical phenomena that can be observed among nanoporous materials. Herein, we have outlined the wide variety of methods our group uses to elucidate their physical and chemical properties, in addition to those of fluids adsorbed in the materials' nanosized pores. We have described simulations at different "levels of theory" and different time and length scales. Computationally demanding simulations are used to investigate complex electronic processes, by explicitly treating the electrons of each framework. Alternatively, larger systems and gas adsorption can be examined by classical potentials that describe atoms or groups of atoms. Finally, we have illustrated statistical physics and geometric approaches that we use to explore porosity and largescale transport. By using this toolbox of methods, in synergy with experimental observations in general, and in situ and in operando characterization in particular, one can obtain a broad and complete picture of the system or process, as well as unparalleled understanding into the microscopic behavior of porous materials.

\section{ASSOCIATED CONTENT}

\section{S Supporting Information}

The Supporting Information is available free of charge on the ACS Publications website at DOI: 10.1021/acs.chemmater.6b02994.

Input files for DFT calculation, $a b$ initio molecular dynamics, and Lattice-Boltzmann simulation (PDF)

\section{AUTHOR INFORMATION}

\section{Corresponding Author}

*E-mail: fx.coudert@chimie-paristech.fr.

\section{Notes}

The authors declare no competing financial interest.

\section{ACKNOWLEDGMENTS}

Although no original calculations were performed in the writing of this review, a large part of the work reviewed here requires access of scientists in the field to large supercomputer centers. We acknowledge GENCI for high-performance computing CPU time allocations (projects x2016087069 and t201608s037). The group's work benefited from the support of ANRT (thèses CIFRE 2015/0268 et 2013/1262), PSL Research University (project DEFORM, grant ANR-10-IDEX0001-02), and the École normale supérieure. F.X.C. thanks Anne Boutin and Alain Fuchs for a long-standing and continuing collaboration on the fascinating topic of modeling of nanoporous materials. J.-M.V. acknowledges Joël Lachambre for the X-ray tomography acquisition and 3D reconstruction performed on the sample shown here. We thank the WOS Bar, Le Bombardier, Picard, and De Clercq for providing moral support in liquid and solid form.

\section{REFERENCES}

(1) Li, J.-R.; Kuppler, R. J.; Zhou, H.-C. Selective gas adsorption and separation in metal-organic frameworks. Chem. Soc. Rev. 2009, 38, 1477.

(2) Morris, R. E.; Wheatley, P. S. Gas Storage in Nanoporous Materials. Angew. Chem., Int. Ed. 2008, 47, 4966-4981.

(3) Bell, A. T. The Impact of Nanoscience on Heterogeneous Catalysis. Science 2003, 299, 1688-1691.

(4) Della Rocca, J. D.; Liu, D.; Lin, W. Nanoscale Metal-Organic Frameworks for Biomedical Imaging and Drug Delivery. Acc. Chem. Res. 2011, 44, 957-968.

(5) Furukawa, H.; Cordova, K. E.; O’Keeffe, M.; Yaghi, O. M. The Chemistry and Applications of Metal-Organic Frameworks. Science 2013, 341, 1230444-1230444.

(6) Davis, M. E. Ordered porous materials for emerging applications. Nature 2002, 417, 813-821.

(7) Zhou, H.-C.; Long, J. R.; Yaghi, O. M. Introduction to MetalOrganic Frameworks. Chem. Rev. 2012, 112, 673-674.

(8) Coudert, F.-X. Responsive Metal-Organic Frameworks and Framework Materials: Under Pressure, Taking the Heat, in the Spotlight, with Friends. Chem. Mater. 2015, 27, 1905-1916.

(9) Sholl, D.; Steckel, J. Density Functional Theory: A Practical Introduction; Wiley: 2011.

(10) Frenkel, D.; Smit, B. Understanding Molecular Simulation, 2nd ed.; Academic Press, Inc.: Orlando, FL, USA, 2001.

(11) Hoover, W. G. Nonequilibrium Molecular Dynamics. Annu. Rev. Phys. Chem. 1983, 34, 103-127.

(12) Hohenberg, P.; Kohn, W. Inhomogeneous Electron Gas. Phys. Rev. 1964, 136, B864-B871.

(13) Kohn, W.; Sham, L. J. Self-Consistent Equations Including Exchange and Correlation Effects. Phys. Rev. 1965, 140, A1133A1138.

(14) Parr, R.; Weitao, Y. Density-Functional Theory of Atoms and Molecules; International Series of Monographs on Chemistry; Oxford University Press: 1994.

(15) Lejaeghere, K.; et al. Reproducibility in density functional theory calculations of solids. Science 2016, 351, aad3000-aad3000.

(16) Dovesi, R.; Orlando, R.; Erba, A.; Zicovich-Wilson, C. M.; Civalleri, B.; Casassa, S.; Maschio, L.; Ferrabone, M.; Pierre, M. D. L.; D’Arco, P.; Noël, Y.; Causà, M.; Rérat, M.; Kirtman, B. C RYSTAL14: A program for the $\mathrm{ab}$ initio investigation of crystalline solids. Int. J. Quantum Chem. 2014, 114, 1287-1317.

(17) Perdew, J. P.; Ruzsinszky, A.; Csonka, G. I.; Vydrov, O. A.; Scuseria, G. E.; Constantin, L. A.; Zhou, X.; Burke, K. Restoring the Density-Gradient Expansion for Exchange in Solids and Surfaces. Phys. Rev. Lett. 2008, 100, 136406.

(18) Thornton, A. W.; Babarao, R.; Jain, A.; Trousselet, F.; Coudert, F.-X. Defects in metalorganic frameworks: a compromise between adsorption and stability? Dalton Trans. 2016, 45, 4352-4359. 
(19) Lee, S. J.; Doussot, C.; Baux, A.; Liu, L.; Jameson, G. B.; Richardson, C.; Pak, J. J.; Trousselet, F.; Coudert, F.-X.; Telfer, S. G. Multicomponent MetalOrganic Frameworks as Defect-Tolerant Materials. Chem. Mater. 2016, 28, 368-375.

(20) Ortiz, A. U.; Boutin, A.; Fuchs, A. H.; Coudert, F.-X. Metalorganic frameworks with wine-rack motif: What determines their flexibility and elastic properties? J. Chem. Phys. 2013, 138, 174703.

(21) Heyd, J.; Scuseria, G. E. Efficient hybrid density functional calculations in solids: Assessment of the Heyd-Scuseria-Ernzerhof screened Coulomb hybrid functional. J. Chem. Phys. 2004, 121, 1187.

(22) Grimme, S. Semiempirical GGA-type density functional constructed with a long-range dispersion correction. J. Comput. Chem. 2006, 27, 1787-1799.

(23) Grimme, S.; Hansen, A.; Brandenburg, J. G.; Bannwarth, C. Dispersion-Corrected Mean-Field Electronic Structure Methods. Chem. Rev. 2016, 116, 5105-5154.

(24) Moellmann, J.; Grimme, S. DFT-D3 Study of Some Molecular Crystals. J. Phys. Chem. C 2014, 118, 7615-7621.

(25) Tkatchenko, A.; DiStasio, R. A.; Car, R.; Scheffler, M. Accurate and Efficient Method for Many-Body van der Waals Interactions. Phys. Rev. Lett. 2012, 108, 236402.

(26) Vanpoucke, D. E. P.; Lejaeghere, K.; Speybroeck, V. V.; Waroquier, M.; Ghysels, A. Mechanical Properties from Periodic Plane Wave Quantum Mechanical Codes: The Challenge of the Flexible Nanoporous MIL-47(V) Framework. J. Phys. Chem. C 2015, 119, 23752-23766.

(27) Barthelet, K.; Marrot, J.; Riou, D.; Férey, G. A Breathing Hybrid Organic-Inorganic Solid with Very Large Pores and High Magnetic Characteristics. Angew. Chem., Int. Ed. 2002, 41, 281-284.

(28) Broyden, C. G. A class of methods for solving nonlinear simultaneous equations. Mathematics of Computation 1965, 19, 577577.

(29) Johnson, D. D. Modified Broyden's method for accelerating convergence in self-consistent calculations. Phys. Rev. B: Condens. Matter Mater. Phys. 1988, 38, 12807-12813.

(30) Reed, A. E.; Weinstock, R. B.; Weinhold, F. Natural population analysisa. J. Chem. Phys. 1985, 83, 735.

(31) Manz, T. A.; Sholl, D. S. Chemically Meaningful Atomic Charges That Reproduce the Electrostatic Potential in Periodic and Nonperiodic Materials. J. Chem. Theory Comput. 2010, 6, 2455-2468.

(32) Gaillac, R.; Pullumbi, P.; Coudert, F.-X. ELATE: an open-source online application for analysis and visualization of elastic tensors. J. Phys.: Condens. Matter 2016, 28, 275201.

(33) Giannozzi, P.; et al. QUANTUM ESPRESSO: a modular and open-source software project for quantum simulations of materials. $J$. Phys.: Condens. Matter 2009, 21, 395502.

(34) Hafner, J. Ab-initio simulations of materials using VASP: Density-functional theory and beyond. J. Comput. Chem. 2008, 29, 2044-2078.

(35) Wills, J. M.; Eriksson, O.; Andersson, P.; Delin, A.; Grechnyev, O.; Alouani, M. Full-Potential Electronic Structure Method; Springer: Berlin Heidelberg, 2010.

(36) Sierka, M.; Sauer, J. Structure and reactivity of silica and zeolite catalysts by a combined quantum mechanics[ndash ]shell-model potential approach based on DFT. Faraday Discuss. 1997, 106, 41-62. (37) Sun, L.; Hendon, C. H.; Minier, M. A.; Walsh, A.; Dincă, M. Million-Fold Electrical Conductivity Enhancement in Fe 2 (DEBDC) versus Mn 2 (DEBDC) (E = S, O). J. Am. Chem. Soc. 2015, 137, 6164-6167.

(38) Odoh, S. O.; Cramer, C. J.; Truhlar, D. G.; Gagliardi, L. Quantum-Chemical Characterization of the Properties and Reactivities of Metal-Organic Frameworks. Chem. Rev. 2015, 115, 6051-6111.

(39) van Speybroeck, V.; Hemelsoet, K.; Joos, L.; Waroquier, M.; Bell, R. G.; Catlow, C. R. A. Advances in theory and their application within the field of zeolite chemistry. Chem. Soc. Rev. 2015, 44, 70447111.
(40) Coudert, F.-X.; Fuchs, A. H. Computational characterization and prediction of metal-organic framework properties. Coord. Chem. Rev. 2016, 307, 211-236.

(41) Marmier, A.; Lethbridge, Z. A.; Walton, R. I.; Smith, C. W.; Parker, S. C.; Evans, K. E. ElAM: A computer program for the analysis and representation of anisotropic elastic properties. Comput. Phys. Commun. 2010, 181, 2102-2115.

(42) Ortiz, A. U.; Boutin, A.; Fuchs, A. H.; Coudert, F.-X. Anisotropic Elastic Properties of Flexible Metal-Organic Frameworks: How Soft are Soft Porous Crystals? Phys. Rev. Lett. 2012, 109, 195502.

(43) Simon, S. H. The Oxford solid state basics; Oxford Univ. Press: Oxford, UK, 2013.

(44) Fultz, B. Vibrational thermodynamics of materials. Prog. Mater. Sci. 2010, 55, 247-352.

(45) Gava, V.; Martinotto, A. L.; Perottoni, C. A. First-Principles Mode Grüneisen Parameters and Negative Thermal Expansion in $\alpha$ ZrW 2 O 8. Phys. Rev. Lett. 2012, 109, 195503.

(46) Cavka, J. H.; Jakobsen, S.; Olsbye, U.; Guillou, N.; Lamberti, C.; Bordiga, S.; Lillerud, K. P. A New Zirconium Inorganic Building Brick Forming Metal Organic Frameworks with Exceptional Stability. J. Am. Chem. Soc. 2008, 130, 13850-13851.

(47) Krause, S.; Bon, V.; Senkovska, I.; Stoeck, U.; Wallacher, D.; Többens, D. M.; Zander, S.; Pillai, R. S.; Maurin, G.; Coudert, F.-X.; Kaskel, S. A pressure-amplifying framework material with negative gas adsorption transitions. Nature 2016, 532, 348-352.

(48) Corradini, D.; Coudert, F.-X.; Vuilleumier, R. Carbon dioxide transport in molten calcium carbonate occurs through an oxoGrotthuss mechanism via a pyrocarbonate anion. Nat. Chem. 2016, 8, 454-460.

(49) Lippert, G.; Hutter, J.; Parrinello, M. The Gaussian and augmented-plane-wave density functional method for ab initio molecular dynamics simulations. Theor. Chem. Acc. 1999, 103, 124140.

(50) VandeVondele, J.; Krack, M.; Mohamed, F.; Parrinello, M.; Chassaing, T.; Hutter, J. Quickstep: Fast and accurate density functional calculations using a mixed Gaussian and plane waves approach. Comput. Phys. Commun. 2005, 167, 103-128.

(51) VandeVondele, J.; Hutter, J. Gaussian basis sets for accurate calculations on molecular systems in gas and condensed phases. J. Chem. Phys. 2007, 127, 114105.

(52) Coudert, F.-X.; Kohen, D. Molecular Insight into $\mathrm{CO}_{2}$ Trapdoor Adsorption in Zeolite Na-RHO. Submitted for publication, 2016.

(53) De Wispelaere, K. D.; Ensing, B.; Ghysels, A.; Meijer, E. J.; Speybroeck, V. V. Complex Reaction Environments and Competing Reaction Mechanisms in Zeolite Catalysis: Insights from Advanced Molecular Dynamics. Chem. - Eur. J. 2015, 21, 9385-9396.

(54) Haigis, V.; Coudert, F.-X.; Vuilleumier, R.; Boutin, A.; Fuchs, A. H. Hydrothermal Breakdown of Flexible Metal-Organic Frameworks: A Study by First-Principles Molecular Dynamics. J. Phys. Chem. Lett. 2015, 6, 4365-4370.

(55) Martinez, M.; Gaigeot, M.-P.; Borgis, D.; Vuilleumier, R Extracting effective normal modes from equilibrium dynamics at finite temperature. J. Chem. Phys. 2006, 125, 144106.

(56) Carter, E.; Ciccotti, G.; Hynes, J. T.; Kapral, R. Constrained reaction coordinate dynamics for the simulation of rare events. Chem. Phys. Lett. 1989, 156, 472-477.

(57) Sprik, M.; Ciccotti, G. Free energy from constrained molecular dynamics. J. Chem. Phys. 1998, 109, 7737.

(58) Haigis, V.; Belkhodja, Y.; Coudert, F.-X.; Vuilleumier, R.; Boutin, A. Challenges in first-principles NPT molecular dynamics of soft porous crystals: A case study on MIL-53(Ga). J. Chem. Phys. 2014, $141,064703$.

(59) Evans, J. D.; Huang, D. M.; Hill, M. R; Sumby, C. J.; Sholl, D. S.; Thornton, A. W.; Doonan, C. J. Molecular Design of Amorphous Porous Organic Cages for Enhanced Gas Storage. J. Phys. Chem. C 2015, 119, 7746-7754.

(60) Evans, J. D.; Coudert, F.-X. Microscopic Mechanism of Chiral Induction in a Metal-Organic Framework. J. Am. Chem. Soc. 2016, $138,6131-6134$. 
(61) Evans, J. D.; Huang, D. M.; Hill, M. R.; Sumby, C. J.; Thornton, A. W.; Doonan, C. J. Feasibility of Mixed Matrix Membrane Gas Separations Employing Porous Organic Cages. J. Phys. Chem. C 2014, $118,1523-1529$.

(62) Trousselet, F.; Boutin, A.; Coudert, F.-X. Novel Porous Polymorphs of Zinc Cyanide with Rich Thermal and Mechanical Behavior. Chem. Mater. 2015, 27, 4422-4430.

(63) Allinger, N. L.; Chen, K.; Lii, J.-H. An improved force field (MM4) for saturated hydrocarbons. J. Comput. Chem. 1996, 17, 642668.

(64) Rappe, A. K.; Casewit, C. J.; Colwell, K. S.; Goddard, W. A.; Skiff, W. M. UFF, a full periodic table force field for molecular mechanics and molecular dynamics simulations. J. Am. Chem. Soc. 1992, 114, 10024-10035.

(65) Cornell, W. D.; Cieplak, P.; Bayly, C. I.; Gould, I. R.; Merz, K. M.; Ferguson, D. M.; Spellmeyer, D. C.; Fox, T.; Caldwell, J. W.; Kollman, P. A. A Second Generation Force Field for the Simulation of Proteins, Nucleic Acids, and Organic Molecules. J. Am. Chem. Soc. 1995, 117, 5179-5197.

(66) Sun, H. COMPASS: An ab Initio Force-Field Optimized for Condensed-Phase ApplicationsOverview with Details on Alkane and Benzene Compounds. J. Phys. Chem. B 1998, 102, 7338-7364.

(67) Getman, R. B.; Bae, Y.-S.; Wilmer, C. E.; Snurr, R. Q. Review and Analysis of Molecular Simulations of Methane, Hydrogen, and Acetylene Storage in Metal-Organic Frameworks. Chem. Rev. 2012, $112,703-723$.

(68) Bureekaew, S.; Amirjalayer, S.; Tafipolsky, M.; Spickermann, C.; Roy, T. K.; Schmid, R. MOF-FF - A flexible first-principles derived force field for metal-organic frameworks. Phys. Status Solidi B 2013, 250, 1128-1141.

(69) Vanduyfhuys, L.; Vandenbrande, S.; Verstraelen, T.; Schmid, R.; Waroquier, M.; van Speybroeck, V. QuickFF: A program for a quick and easy derivation of force fields for metal-organic frameworks from ab initio input. J. Comput. Chem. 2015, 36, 1015-1027.

(70) Wieme, J.; Vanduyfhuys, L.; Rogge, S. M. J.; Waroquier, M.; van Speybroeck, V. Exploring the Flexibility of MIL-47(V)-Type Materials Using Force Field Molecular Dynamics Simulations. J. Phys. Chem. C 2016, 120, 14934-14947.

(71) Addicoat, M. A.; Vankova, N.; Akter, I. F.; Heine, T. Extension of the Universal Force Field to Metal-Organic Frameworks. J. Chem. Theory Comput. 2014, 10, 880-891.

(72) Fogarty, A. C.; Coudert, F.-X.; Boutin, A.; Laage, D. Reorientational Dynamics of Water Confined in Zeolites. ChemPhysChem 2014, 15, 521-529.

(73) Fincham, D. Choice of timestep in molecular dynamics simulation. Comput. Phys. Commun. 1986, 40, 263-269.

(74) Hünenberger, P. H. Advanced Computer Simulation; Springer Science + Business Media: 2005; pp 105-149.

(75) Rogge, S.; Vanduyfhuys, L.; Ghysels, A.; Waroquier, M.; Verstraelen, T.; Maurin, G.; Speybroeck, V. V. A Comparison of Barostats for the Mechanical Characterization of Metal-Organic Frameworks. J. Chem. Theory Comput. 2015, 11, 5583-5597.

(76) Nosé, S. A unified formulation of the constant temperature molecular dynamics methods. J. Chem. Phys. 1984, 81, 511.

(77) Hoover, W. G. Canonical dynamics: Equilibrium phase-space distributions. Phys. Rev. A: At., Mol., Opt. Phys. 1985, 31, 1695-1697.

(78) Martyna, G. J.; Tobias, D. J.; Klein, M. L. Constant pressure molecular dynamics algorithms. J. Chem. Phys. 1994, 101, 4177.

(79) Dubbeldam, D.; Torres-Knoop, A.; Walton, K. S. On the inner workings of Monte Carlo codes. Mol. Simul. 2013, 39, 1253-1292.

(80) HASTINGS, W. K. Monte Carlo sampling methods using Markov chains and their applications. Biometrika 1970, 57, 97-109.

(81) Bouëssel du Bourg, L. B.; Ortiz, A. U.; Boutin, A.; Coudert, F.-X. Thermal and mechanical stability of zeolitic imidazolate frameworks polymorphs. APL Mater. 2014, 2, 124110.

(82) Plimpton, S. Fast Parallel Algorithms for Short-Range Molecular Dynamics. J. Comput. Phys. 1995, 117, 1-19.

(83) Abraham, M. J.; Murtola, T.; Schulz, R.; Páll, S.; Smith, J. C.; Hess, B.; Lindahl, E. GROMACS: High performance molecular simulations through multi-level parallelism from laptops to supercomputers. SoftwareX 2015, 1-2, 19-25.

(84) Smith, W.; Todorov, I. T. A short description of DL_POLY. Mol. Simul. 2006, 32, 935-943.

(85) Dubbeldam, D.; Calero, S.; Ellis, D. E.; Snurr, R. Q. RASPA: molecular simulation software for adsorption and diffusion in flexible nanoporous materials. Mol. Simul. 2016, 42, 81-101.

(86) Design, M. MedeA GIBBS: Thermodynamics of fluids and adsorption. http://www.materialsdesign.com/medea/gibbs, 2016.

(87) Triguero, C.; Coudert, F.-X.; Boutin, A.; Fuchs, A. H.; Neimark, A. V. Mechanism of Breathing Transitions in Metal-Organic Frameworks. J. Phys. Chem. Lett. 2011, 2, 2033-2037.

(88) Coudert, F.-X.; Mellot-Draznieks, C.; Fuchs, A. H.; Boutin, A. Prediction of Breathing and Gate-Opening Transitions Upon Binary Mixture Adsorption in Metal-Organic Frameworks. J. Am. Chem. Soc. 2009, 131, 11329-11331.

(89) Coudert, F.-X.; Jeffroy, M.; Fuchs, A. H.; Boutin, A.; MellotDraznieks, C. Thermodynamics of Guest-Induced Structural Transitions in Hybrid Organic-Inorganic Frameworks. J. Am. Chem. Soc. 2008, 130, 14294-14302.

(90) Eslami, H.; Müller-Plathe, F. Molecular dynamics simulation in the grand canonical ensemble. J. Comput. Chem. 2007, 28, 1763-1773.

(91) Kuznetsova, T.; Kvamme, B. Grand canonical molecular dynamics for TIP4P water systems. Mol. Phys. 1999, 97, 423-431.

(92) Gupta, A.; Chempath, S.; Sanborn, M. J.; Clark, L. A.; Snurr, R. Q. Object-oriented Programming Paradigms for Molecular Modeling. Mol. Simul. 2003, 29, 29-46.

(93) Darden, T.; York, D.; Pedersen, L. Particle mesh Ewald: An N. $\log (\mathrm{N})$ method for Ewald sums in large systems. J. Chem. Phys. 1993, 98, 10089.

(94) Fukuda, I.; Yonezawa, Y.; Nakamura, H. Consistent Molecular Dynamics Scheme Applying the Wolf Summation for Calculating Electrostatic Interaction of Particles. J. Phys. Soc. Jpn. 2008, 77, 114301.

(95) Soergel, D. A. W. Rampant software errors may undermine scientific results. F1000Research 2015, 303.

(96) Wilson, G.; Aruliah, D. A.; Brown, C. T.; Hong, N. P. C.; Davis, M.; Guy, R. T.; Haddock, S. H. D.; Huff, K. D.; Mitchell, I. M.; Plumbley, M. D.; Waugh, B.; White, E. P.; Wilson, P. Practices for Scientific Computing. PLoS Biol. 2014, 12, e1001745.

(97) Torvalds, L.; Hamano, J. Git. http://www.git-scm.com, 2016.

(98) Preston-Wernerm, T.; Wanstrath, C.; Hyett, P. Github. http:// www.github.com, 2016.

(99) Fuchs, S. Travis CI. http://www.travis-ci.com, 2016.

(100) Bae, Y.-S.; Snurr, R. Q. Development and Evaluation of Porous Materials for Carbon Dioxide Separation and Capture. Angew. Chem., Int. Ed. 2011, 50, 11586-11596.

(101) Farha, O. K.; Eryazici, I.; Jeong, N. C.; Hauser, B. G.; Wilmer, C. E.; Sarjeant, A. A.; Snurr, R. Q.; Nguyen, S. T.; Özgür Yazaydın, A.; Hupp, J. T. Metal-Organic Framework Materials with Ultrahigh Surface Areas: Is the Sky the Limit? J. Am. Chem. Soc. 2012, 134, 15016-15021.

(102) Brunauer, S.; Emmett, P. H.; Teller, E. Adsorption of Gases in Multimolecular Layers. J. Am. Chem. Soc. 1938, 60, 309-319.

(103) Rouquerol, J.; Llewellyn, P.; Rouquerol, F. Stud. Surf. Sci. Catal.; Elsevier BV: 2007; pp 49-56.

(104) Wang, T. C.; Bury, W.; Gómez-Gualdrón, D. A.; Vermeulen, N. A.; Mondloch, J. E.; Deria, P.; Zhang, K.; Moghadam, P. Z.; Sarjeant, A. A.; Snurr, R. Q.; Stoddart, J. F.; Hupp, J. T.; Farha, O. K. Ultrahigh Surface Area Zirconium MOFs and Insights into the Applicability of the BET Theory. J. Am. Chem. Soc. 2015, 137, 35853591.

(105) Sarkisov, L.; Kim, J. Computational structure characterization tools for the era of material informatics. Chem. Eng. Sci. 2015, 121, $322-330$.

(106) Sarkisov, L.; Harrison, A. Computational structure characterisation tools in application to ordered and disordered porous materials. Mol. Simul. 2011, 37, 1248-1257. 
(107) First, E. L.; Gounaris, C. E.; Wei, J.; Floudas, C. A. Computational characterization of zeolite porous networks: an automated approach. Phys. Chem. Chem. Phys. 2011, 13, 17339.

(108) Willems, T. F.; Rycroft, C. H.; Kazi, M.; Meza, J. C.; Haranczyk, M. Microporous Mesoporous Mater. 2012, 149, 134-141.

(109) Levesque, M.; Duvail, M.; Pagonabarraga, I.; Frenkel, D.; Rotenberg, B. Accounting for adsorption and desorption in lattice Boltzmann simulations. Phys. Rev. E 2013, 88, 013308.

(110) Ladd, A. J. C.; Verberg, R. Lattice-Boltzmann Simulations of Particle-Fluid Suspensions. J. Stat. Phys. 2001, 104, 1191-1251.

(111) Frisch, U.; d'Humières, D.; Hasslacher, B.; Lallemand, P.; Pomeau, Y.; Rivet, J.-P. Lattice gas hydrodynamics in two and three dimensions. Complex Systems 1987, 1, 649-707.

(112) d'Humières, D.; Lallemand, P.; Frisch, U. Lattice gas model for 3D hydrodynamics. Europhys. Lett. 1986, 2, 291-297.

(113) Frisch, U.; Hasslacher, B.; Pomeau, Y. Lattice-Gas Automata for the Navier-Stokes equation. Phys. Rev. Lett. 1986, 56, 1505-1509.

(114) Mei, R.; Shyy, W.; Yu, D.; Luo, L.-S. Lattice Boltzmann Method for 3-D Flows with Curved Boundary. J. Comput. Phys. 2000, 161, 680-699.

(115) Succi, S. The Lattice-Boltzmann equation for fluid dynamics and beyond; Oxford Science Publications: 2001.

(116) Lowe, C.; Frenkel, D. The super long-time decay of velocity fluctuations in a two-dimensional fluid. Phys. A 1995, 220, 251-260.

(117) Lowe, C. P.; Frenkel, D. Do Hydrodynamic Dispersion Coefficients Exist? Phys. Rev. Lett. 1996, 77, 4552-4555.

(118) Vanson, J.-M.; Coudert, F.-X.; Rotenberg, B.; Levesque, M.; Tardivat, C.; Klotz, M.; Boutin, A. Unexpected coupling between flow and adsorption in porous media. Soft Matter 2015, 11, 6125-6133. 